\title{
EXAFS studies of rare-earth metaphosphate glasses
}

\begin{abstract}
An extended x-ray-absorption fine structure (EXAFS) study has been carried out on a range of rare-earth metaphosphate $\mathrm{R}(\mathrm{PO} 3) 3$ glasses of growing interest in optical communications and laser technologies. Phosphate glasses modified using the rare-earth oxides Pr6O11, $\mathrm{Nd} 2 \mathrm{O} 3, \mathrm{Eu} 2 \mathrm{O} 3, \mathrm{Gd} 2 \mathrm{O} 3, \mathrm{~Tb} 2 \mathrm{O} 3$, and $\mathrm{Ho} 2 \mathrm{O} 3$, have been investigated using their respective rare-earth LIII absorption edges. The data provide information on the local environment of the rare-earth ion within the phosphate glass matrix constructed from linked PO4 tetrahedra. The rare-earth ions occupy sites with an average coordination number in the range, 6ÒNÒ, the surrounding atoms being oxygen. The first shell interatomic distance over the range of rare-earth ions establishes the rare-earth contraction of ionic radii with increasing atomic number in a series of glasses. There is also evidence for a rare-earth-phosphorus correlation between 2.7 and $3.6 \AA$, and a further rare-earth-oxygen correlation at approximately $4 \AA$. The EXAFS spectrum shows no evidence for R-R correlations within the short-range order, a result especially pertinent to the optical and magnetic properties of the glasses. The fractal dimensionality 4C11/B of these glasses, obtained from the elastic stiffnesses determined from ultrasonic wave velocities, ranges between 2.3 and 2.8, indicating that their connectivity tends towards having a three-dimensional character.
\end{abstract}

Keyword: Extended x-ray-absorption fine structure (EXAFS); Rare-earth; Metaphosphate glasses 